\title{
Ratio Between Lactobacillus Plantarum and Acetobacter Pomorum on the Surface of Drosophila Melanogaster Adult Flies Depends on Cuticle Melanisation
}

\author{
Vladislav Mokeev \\ University of Tübingen: Eberhard Karls Universitat Tubingen \\ Yiwen Wang \\ Tianjin University \\ Nicole Gehring \\ University of Tübingen: Eberhard Karls Universitat Tubingen \\ Bernard Moussian ( $\sim$ bernard.moussian@unice.fr ) \\ Université Nice Sophia Antipolis https://orcid.org/0000-0002-2854-9500
}

Research note

Keywords: Microbiome, bacteria, insect, Drosophila, cuticle

Posted Date: May 18th, 2021

DOl: https://doi.org/10.21203/rs.3.rs-523605/v1

License: (c) (i) This work is licensed under a Creative Commons Attribution 4.0 International License.

Read Full License 

on the surface of Drosophila melanogaster adult flies depends on cuticle melanisation

3 Vladislav Mokeev ${ }^{1}$, Yiwen Wang ${ }^{1,2}$, Nicole Gerhring ${ }^{1} \&$ Bernard Moussian $^{1,3^{*}}$

41 University of Tübingen, Interfaculty Institute of Cell Biology, Section Animal Genetics, Auf 5 der Morgenstelle 15, 72076 Tübingen, Germany

62 School of Pharmaceutical Science and Technology, Tianjin University, 300072, Tianjin, 7 China

83 Université Côte d'Azur, Parc Valrose, 06108 Nice CEDEX 2, France

9 * Correspondence: bernard.moussian@unice.fr

\section{Abstract}

11 Objectives

12 As in most organisms, the surface of the fruit fly Drosophila melanogaster is associated with 13 bacteria. In order to study the genetic parameters of this association, we developed a simple 14 protocol for surface bacteria isolation and quantification.

15 Results

16 On wild-type flies maintained in the laboratory, we identified two persistently culturable 17 species as Lactobacillus plantarum and Acetobacter pomorum by $16 \mathrm{~S}$ rDNA sequencing. For 18 quantification, we showered single flies for DNA extraction avoiding the rectum to prevent contamination from the gut. Using specific primers for quantitative PCR analyses, we determined the relative abundance of these two species in surface wash samples. Repeatedly, 21 we found $20 \%$ more L. plantarum than A. pomorum. To tentatively study the importance of the 22 cuticle for the interaction of the surface with these bacteria, applying Crispr/Cas9 gene editing 23 in the initial wild-type flies, we generated flies mutant for the ebony gene needed for cuticle 24 melanisation and determined the L. plantarum to A. pomorum ratio on these flies. We found 25 that the relative abundance of $L$. plantarum increased substantially on ebony flies. We conclude 
that the cuticle chemistry is crucial for surface bacteria composition. This finding may inspire future studies on cuticle-microbiome interactions.

\section{Keywords}

Microbiome, bacteria, insect, Drosophila, cuticle

\section{Introduction}

Bacteria populate the surface of many organisms. While skin bacteria are well analysed in vertebrates including humans [1,2], surface bacteria-insect interactions have been largely neglected to date. Most of data on surface bacteria come from studies in ants such as Camponotus femoratus and Crematogaster levior. In ant colonies, surface bacteria are considered to be involved in protection against fungal infection [3, 4]. A few data are available on bacteria on the surface of the fruit fly Drosophila melanogaster. The most common surface bacteria in this species belong to the genera Lactobacillus and Acetobacter [5]. The role of surface bacteria in D. melanogaster has not been studied.

The parameters on the insect defining bacteria-insect surface association are largely unknown.

It is conceivable that microorganisms interact with components of the cuticle that is a stratified extracellular matrix composed of chitin, proteins, catecholamines and lipids [6]. Especially, the components of the surface called envelope including waxes and cuticular hydrocarbons (CHCs) [7] may be used as a substrate for bacterial attachment and/or for nutrition. In addition, this interaction may also depend on the inner-cuticle chemical environment including water content that in turn, at least partly, depends on the hardening and melanisation degree of the cuticle that involves a well-studied cascade of reactions catalised by cytoplasmic and extracellular enzymes [8].

In the present work, we have designed a protocol for surface bacteria isolation and relative quantification in D. melanogaster. In a pilot experiment, we show that the bacterial composition depends on cuticle melanisation. 


\section{Materials and Methods}

Fly work

Wild-type Tübingen 2018 flies were kept under laboratory conditions $\left(22^{\circ} \mathrm{C}, 50-70 \%\right.$ air humidity) in vials with artificial diet that consists of corn meal, agar, beet sugar, propionic acid, dry yeast and Nipagin M. For fluorescein feeding, fluorescein sodium salt (Sigma Aldrich) was mixed with fresh baker's yeast added to the vials.

\section{Isolation of surface bacteria}

Flies were individually rubbed against the surface of a sterile agar plate (China Blue, ECI, EMB, LB, BHI and MRS, ingredients from Sigma-Aldrich) inside laminar conditions using sterile forceps forming a short lane and incubated for 1-5 days aerobically. Cultivation condition for each media were chosen depending on used media. We obtained mixed populations of different microorganisms. Individual colonies were isolated and sub-cultured twice to ensure purity. Isolated bacteria were characterized morphologically using a light microscopy and identified using a 16S rDNA analysis.

\section{Molecular biology}

The DNA template was prepared by picking an individual colony of each bacterial strain. Amplification of the 16S rDNA gene was carried out according to a standard protocol by PCR using the universal primers 27F (5'-AGAGTTTGATCCTGGCTCAG-3') and 1492R (5'GGTTACCTTGTTACGACTT-3') [9]. PCR products were purified before sequencing using the GenElute ${ }^{\mathrm{TM}}$ PCR Clean-Up Kit (Sigma-Aldrich). To identify the species, the sequences were aligned to sequences of the NCBI database using BlastN.

For species-specific quantitative PCR (qPCR) experiments, single flies were immobilised with forceps and spilled with Tris-EDTA (pH8.0) containing 200ng/ $\mu 1$ Proteinase K avoiding the rectum. The wash solutions of 20 flies were combined, incubated at $65^{\circ} \mathrm{C}$ for 30 minutes and 
frozen at $-20^{\circ} \mathrm{C}$. After thawing and centrifugation, $5 \mu$ of this solution was used in a $10 \mu 1$ reaction solution containing $1 \mu 1$ of each species-specific primer. For species-specific qPCR, the

primers pREV

(5'-TCGGGATTACCAAACATCAC-3')

and

$\mathrm{pLanF}$

$\left(5^{\prime}-\right.$

CCGTTTCTGCGGAACACCTA-3') to amplify recA (318 bp) in Lactobacillus plantarum [10] and PASTEU-F (5'-TCAAGTCCTCATGGCCCTTATG-3') and PASTEU-R (5'TCGAGTTGCAGAGTGCAATCC-3') to amplify 130 bp of the 16S rDNA loci of Acetobacter species including A. pomorum and pasteurianus were used [11].

To mutate the ebony locus in Tübingen 2018 flies, gene editing according to the Crispr/cas9 method was applied. We used the published ebony gDNA (oligos: 5'GCGTTTAGTCGCAAAGAAGAA-3' and 5'-TACTGCCCGAGGTGTAGAGC-3') directed against the ebony gene sub-cloned in the pCDF3 vector [12]. This construct $(550 \mathrm{ng} / \mu 1 \mathrm{TE}$ buffer) was injected into pre-blastoderm embryos together with $250 \mathrm{ng} / \mu 1$ of Cas 9 protein (New England Biolabs). To identify mutant ebony alleles, the respective flies were crossed to flies segregating the TM6B balancer that carries the ebony ${ }^{l}$ allele. Stocks of dark flies were established. Homozygous ebony mutant flies (ebony ccl or $^{3}$ ) were sequenced to identify the mutation.

\section{Microscopy}

Bacterial colonies were observed and imaged on a Leica EZ4 stereomicroscope with in-built camera using the software LAX. Bacterial cells were viewed on a Nikon Ti2 microscope using phase contrast microscopy with a S Plan Fluor ELWD 40x Ph2 ADM objective.

To visualize fluorescein traces on the fly surface, flies were anesthetized with $\mathrm{CO}_{2}$ and viewed with the Nikon AZ100 using fluorescence microscopy mode with a LED light source and a F36-525 HC-set EGFP filter. 


\section{Results and discussion}

100

101

102

103

104

105

106

107

108

109

110 fly surface

111 To isolate and characterise fly surface bacteria, we streaked the dorsum of living wild-type flies

112 from Tübingen on different media including MRS (DeMan, Rogosa, Sharpe). To exclude gut-

113 derived bacteria, we avoided contacting the rectum with the medium. Persistently, in

114 independent experiments, we observed two types of colonies on MRS plates (Fig. 1). The

115 colonies were round and white or yellowish. Under the light microscope, bacteria from both

116 colonies showed a rode shape (Fig. 1). To determine the species, we amplified the 16S rDNA

117 locus using universal primers and sequenced the amplicon. Alignment of the amplified

118 sequences with sequences from the NCBI nucleotide database revealed that the 16S rDNA

119 sequence from bacteria forming white colonies was highly similar to the respective sequence

120 from Lactobacillus plantarum (Table 1), while the 16S rDNA sequence from bacteria forming

121 yellow colonies was highly similar to the respective sequence from Acetobacter species including pomorum and pasteurianus (Table 1). To distinguish between these two species, we determined the sequence of the groEL gene. The groEL sequence amplified from our bacteria 
124 was more similar to the groEL sequence of $A$. pomorum than to the respective sequence of $A$. pasteurianus.

These two species had been found to be present in the D. melanogaster gut [14]. In the gut, $L$. plantarum was reported to promote growth by interfering with the insulin and ecdysone signalling pathways on poor-condition medium [15]. In another work, it was found that, by contrast, intestinal L. plantarum had a negative effect on D. melanogaster life span [16]. We presume that $L$. plantarum on the cuticle surface does not contribute to any of these effects as the gut and surface micro-environments are fundamentally different. Indeed, while in the gut these bacteria live under anaerobic conditions, on the fly surface, they rather face aerobic conditions. Supposedly, their physiology and, by consequence, their role changes accordingly. For instance, aerobic but not anaerobic cultures of L. plantarum produce $\mathrm{H}_{2} \mathrm{O}_{2}$ [17]. At the cuticle surface, $\mathrm{H}_{2} \mathrm{O}_{2}$ might oxidise cuticular hydrocarbons (CHCs) and thereby modify the barrier function of this layer. A second possible function of L. plantarum on the cuticle surface is attraction of partners and to promote crowding. Indeed, D. melanogaster has been shown to be attracted by yet unidentified volatile compounds of L. plantarum [18].

The fly surface is not soiled by faeces

L. plantarum and A. pomorum are also present in the gut suggesting that their presence on the fly surface may originate from faeces. To verify whether the surface of flies contains excreted material, we fed adult flies with fluorescein and imaged their surface by fluorescence microscopy (Fig. S1). Only very little fluorescence signal was detected on the fly surface. We conclude that, overall, contamination of the surface by faeces is negligible.

\section{Relative quantification of bacteria by $q P C R$}

Isolation and cultivation of bacteria from the fly surface on media plates does not allow relative quantification as standardisation of bacterial transfer from flies to the plate is not possible. Therefore, we determined the ratio between L. plantarum and A. pomorum indirectly using species-specific primers in quantitative PCR (qPCR) experiments. We first compared $L$. 
plantarum and A. pomorum abundance on Tübingen 2018 flies (Fig. 2). Approximately, there were $20 \%$ more $L$. plantarum than A. pomorum on the surface of Tübingen 2018 flies.

In order to test the influence of the cuticle on the load of L. plantarum and A. pomorum, by gene editing, we introduced mutations in the ebony gene of Tübingen 2018 flies that codes for $\beta$-alanyl-dopamine (NBAD) synthase involved in the cuticle melanisation pathway [8]. Three independent mutations in the ebony gene (ebony $y^{c c 1}$, ebony ${ }^{c c 3}$ and $e b o n y^{c c 4}$ ) were recovered. The respective homozygous mutant flies that are darker than wild-type flies are viable. We

157 determined the ratio between L. plantarum and A. pomorum on the surface of ebony ${ }^{c c l}$ and $158 e b o n y^{c c 3}$ flies by qPCR (Fig. 2). We found that compared to Tübingen 2018 control flies, the 159 ratio between L. plantarum and A. pomorum on the surface of ebony flies was enhanced. We conclude that the L. plantarum and A. pomorum load depends on Ebony and probably on melanisation. It remains to be shown whether Ebony and melanisation either promote $L$. plantarum or inhibit A. pomorum growth. Classically, according to the melanism-desiccation

163 hypothesis, enhanced melanisation has been considered as a response to dry environment to 164 prevent desiccation [7]. For instance, in the melanic drosophilid D. kikkawai higher abdominal melanisation correlates with enhanced desiccation resistance [19]. However, there are cases reported that contradict this hypothesis [20, 21]. Desiccation resistance, for example, did not

167 correlate with the body colour intensity in D. melanogaster wild populations in India [21]. We 168 conclude that melanisation is probably a trade-off trait not only dictated by humidity conditions.

169 Based on this assumption, we speculate that Ebony-driven melanisation may also be involved 170 in controlling the interaction between the fly body and bacteria conferring a yet unknown 171 advantage. Alternatively, Ebony may have a direct or indirect function in the differentiation of 172 the envelope and the surface CHCs. Indeed, in a recent work, it was found that longer chain 173 CHCs prevailed in ebony mutant females [22]. This suggests that L. plantarum and A. pomorum 174 differ in their preference on CHC environment. In summary, these data support the view that 175 the insect cuticle surface is not an inert substrate for bacteria. 


\section{Limitations}

177 The ratio between L. plantarum and A. pomorum on the surface of D. melanogaster changes

178 depending on the genetic background suggesting that the insect-bacteria interaction may be

179 under genetic control. The significance of this interaction is unclear as our conclusion relies

180 only on the impact of a single gene i.e. ebony on the insect-bacteria interaction. More work is

181 needed in this direction.

182 We should also point out that the flies used in this work were kept under laboratory conditions.

183 Hence, it is unclear whether our work reflects the situation in the field.

184 A major uncertainty in this work concerns the bacterial species. The amplified A. pomorum 16S

185 rDNA sequence is $100 \%$ identical to the respective sequence in A. pasteurianus [11]. The

186 provisional identification $A$. pomorum is based on the groEL sequence. The groEL sequence

187 determined in this work is, however, not identical to the A. pomorum groEL sequence from the

188 NCBI database. Thus, it is well possible that the Acetobacter species isolated in this work is

189 neither pomorum nor pasteurianus but a third yet unknown species not present in the sequence

190 databases. Additional analyses are needed to clarify this issue.

\section{Declarations}

192 List of abbreviations

193 Apom: Acetobacter pomorum

194 bp: base pairs

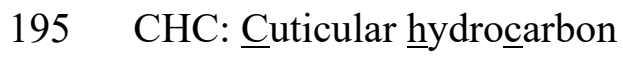

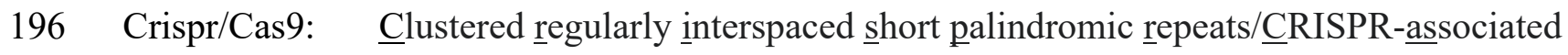

197 protein $\underline{9}$

198 gDNA: guide DNA

199 groEL: $60 \mathrm{kDa}$ chaperonin

200 Lpla: Lactobacillus planatrum 
201 qPCR: quantitative polymerase chain reaction

202 16S rDNA: 16S ribosomal DNA

203 Ethics approval and consent to participate

204 Not applicable.

205 Consent to publish

206 Not applicable.

207 Availability of data and materials

208 All data are presented in the manuscript. Upon request, fly stocks will be shared by the

209 corresponding author.

210 Competing interests

211 The authors declare that they have no competing interests.

$212 \quad$ Funding

213 Funding for this work was provided by the German Research Foundation to B.M. (MO1714/9-

214 1, University of Tübingen). The funders had no role in study design, experiment execution or 215 analysis, decision to publish, or preparation of this manuscript.

216 Authors' contributions

217 VM, NG and BM carried out the molecular biology. NG and BM generated CRISPR/Cas9218 edited fly strains. VM and BM executed the analysis. SJM wrote the initial draft of the 219 manuscript. VM. YW and BM interpreted the data. BM finalized the manuscript. All authors 220 read and approved the final manuscript.

221 Corresponding author

222 Correspondence to Bernard Moussian.

223 Acknowledgements

224 Not applicable. 
226 1. Schommer NN, Gallo RL: Structure and function of the human skin microbiome. Trends Microbiol 2013, 21(12):660-668.

2. Chen YE, Fischbach MA, Belkaid Y: Skin microbiota-host interactions. Nature 2018, 553(7689):427-436.

3. Birer C, Moreau CS, Tysklind N, Zinger L, Duplais C: Disentangling the assembly mechanisms of ant cuticular bacterial communities of two Amazonian ant species sharing a common arboreal nest. Mol Ecol 2020, 29(7):1372-1385.

4. Mattoso TC, Moreira DD, Samuels RI: Symbiotic bacteria on the cuticle of the leaf-cutting ant Acromyrmex subterraneus subterraneus protect workers from attack by entomopathogenic fungi. Biol Lett 2012, 8(3):461464.

5. Ren C, Webster P, Finkel SE, Tower J: Increased internal and external bacterial load during Drosophila aging without life-span trade-off. Cell Metab 2007, 6(2):144-152.

6. Moussian B: Recent advances in understanding mechanisms of insect cuticle differentiation. Insect Biochemistry and Molecular Biology 2010, 40(5):363-375.

7. Wang Y, Ferveur JF, Moussian B: Eco-genetics of desiccation resistance in Drosophila. Biol Rev Camb Philos Soc 2021.

8. Noh MY, Muthukrishnan S, Kramer KJ, Arakane Y: Cuticle formation and pigmentation in beetles. Curr Opin Insect Sci 2016, 17:1-9.

9. Janda JM, Abbott SL: 16S rRNA gene sequencing for bacterial identification in the diagnostic laboratory: pluses, perils, and pitfalls. $J$ Clin Microbiol 2007, 45(9):2761-2764. 
251 10. Tsai C-C, Lai C-H, Yu B, Tsen H-Y: Use of PCR primers and probes based on the 23S rRNA and internal transcription spacer (ITS) gene sequence for the detection and enumerization of Lactobacillus acidophilus and Lactobacillus plantarum in feed supplements. Anaerobe 2010, 16:270-277.

11. Torija MJ, Mateo E, Guillamon JM, Mas A: Identification and quantification of acetic acid bacteria in wine and vinegar by TaqMan-MGB probes. Food Microbiol 2010, 27(2):257-265.

12. Port F, Chen HM, Lee T, Bullock SL: Optimized CRISPR/Cas tools for efficient germline and somatic genome engineering in Drosophila. Proc Natl Acad Sci U S A 2014, 111(29):E2967-2976.

13. Birer C, Tysklind N, Zinger L, Duplais C: Comparative analysis of DNA extraction methods to study the body surface microbiota of insects: A case study with ant cuticular bacteria. Mol Ecol Resour 2017, 17(6):e34-e45.

14. Broderick NA, Lemaitre B: Gut-associated microbes of Drosophila melanogaster. Gut Microbes 2012, 3(4):307-321.

15. Storelli G, Defaye A, Erkosar B, Hols P, Royet J, Leulier F: Lactobacillus plantarum promotes Drosophila systemic growth by modulating hormonal signals through TOR-dependent nutrient sensing. Cell Metab 2011, 14(3):403-414.

16. Fast D, Duggal A, Foley E: Monoassociation with Lactobacillus plantarum Disrupts Intestinal Homeostasis in Adult Drosophila melanogaster. mBio 2018, 9(4). peroxide accumulation with oxygen induced enzymes in Lactobacillus plantarum cultures. Arch Microbiol 1984, 138(1):44-48. 
18. Qiao H, Keesey IW, Hansson BS, Knaden M: Gut microbiota affects development and olfactory behavior in Drosophila melanogaster. $J$ Exp Biol 2019, 222(Pt 5).

19. Ramniwas S, Kajla B: Divergent strategy for adaptation to drought stress in two sibling species of montium species subgroup: Drosophila kikkawai and Drosophila leontia. J Insect Physiol 2012, 58(12):1525-1533.

20. Rajpurohit S, Peterson LM, Orr AJ, Marlon AJ, Gibbs AG: An Experimental Evolution Test of the Relationship between Melanism and Desiccation Survival in Insects. PLoS One 2016, 11(9):e0163414.

22. Massey JH, Akiyama N, Bien T, Dreisewerd K, Wittkopp PJ, Yew JY, Takahashi Hydrocarbon Composition in Drosophila melanogaster. Front Physiol 2019, 10:518.

Tables

294 Table 1. Identification of the bacterial species.

\begin{tabular}{|c|c|c|c|}
\hline locus & Sequence length & identity & species \\
\hline $16 S \mathrm{SDNA}$ & $853 \mathrm{bp}$ & $98,25 \%$ & Lactobacillus plantarum \\
\hline $16 \mathrm{~S} r \mathrm{DNA}$ & $982 \mathrm{bp}$ & $98,02 \%$ & Lactobacillus pentosus \\
\hline & & $99,80 \%$ & Lactobacillus plantarum \\
\hline $16 \mathrm{~S} r \mathrm{DNA}$ & $940 \mathrm{bp}$ & $99,25 \%$ & Lactobacillus pentosus \\
\hline
\end{tabular}




\begin{tabular}{|c|c|c|c|}
\hline & & $99,25 \%$ & Acetobacter pasteurianus \\
\hline $16 S \mathrm{rDNA}$ & $1050 \mathrm{bp}$ & $100 \%$ & Acetobacter pomorum \\
\hline groEL & $560 \mathrm{bp}$ & $100 \%$ & Acetobacter pasteurianus \\
\hline & & $98,57 \%$ & Acetobacter pomorum \\
\hline
\end{tabular}

295 The sequence of the 16S rDNA locus suggests that the white colonies shown in Figure 1 are $L$.

296 plantarum. This sequence is not sufficient to determine the species of the beige colonies. Based

297 on the groEL sequence, we assume that these bacteria are $A$. pomorum.

\section{Figure legends}

299 Figure 1. L. plantarum and A. pomorum are present on the surface of D. melanogaster.

300 Upon streaking D. melanogaster on a plate, two types of heaps of bacteria were observed (A).

301 We isolated single colonies that were white or beige (B). After sequencing the 16S rDNA, the

302 white colonies were identified as L. plantarum (Lpla) and the beige colonies as A. pomorum

303 (Apom). Under the microscope, both bacteria are a rode shaped (L. plantarum, C, A. pomorum, 304 D).

305 Figure 2. The ratio between L. plantarum and A. pomorum depends on the fly genotype.

306 Applying qPCR, we detected L. plantarum and A. pomorum in the wash solution of fly surfaces 307 in three independent experiments (1-3). In ebony mutant flies (ebony $\left.y^{c c 1} \& 3\right)$ that derive from 308 Tübingen 2018 flies (Tü 2018) by gene editing (Crispr/Cas9), the L. plantarum to A. pomorum 309 ratio is higher than in the original flies. The expression levels on Tübingen 2018 flies are set to 310 one. Statistical analyses are not useful as the expression changes in the different experiments 311 are enormous, although a clear trend is obvious.

\section{Supplementary information}

313 Additional file

314 Figure S1. There are only little faeces on the fly surface. 
315 The surface of flies fed with yeast supplemented with fluorescein did not show abundant 316 fluorescence signal (arrows). 
Figure 1

A

$0.5 \mathrm{~mm}$
B

Apom

\section{Lpla}

$0.5 \mathrm{~mm}$ 
Figure 2 


\section{Figures}

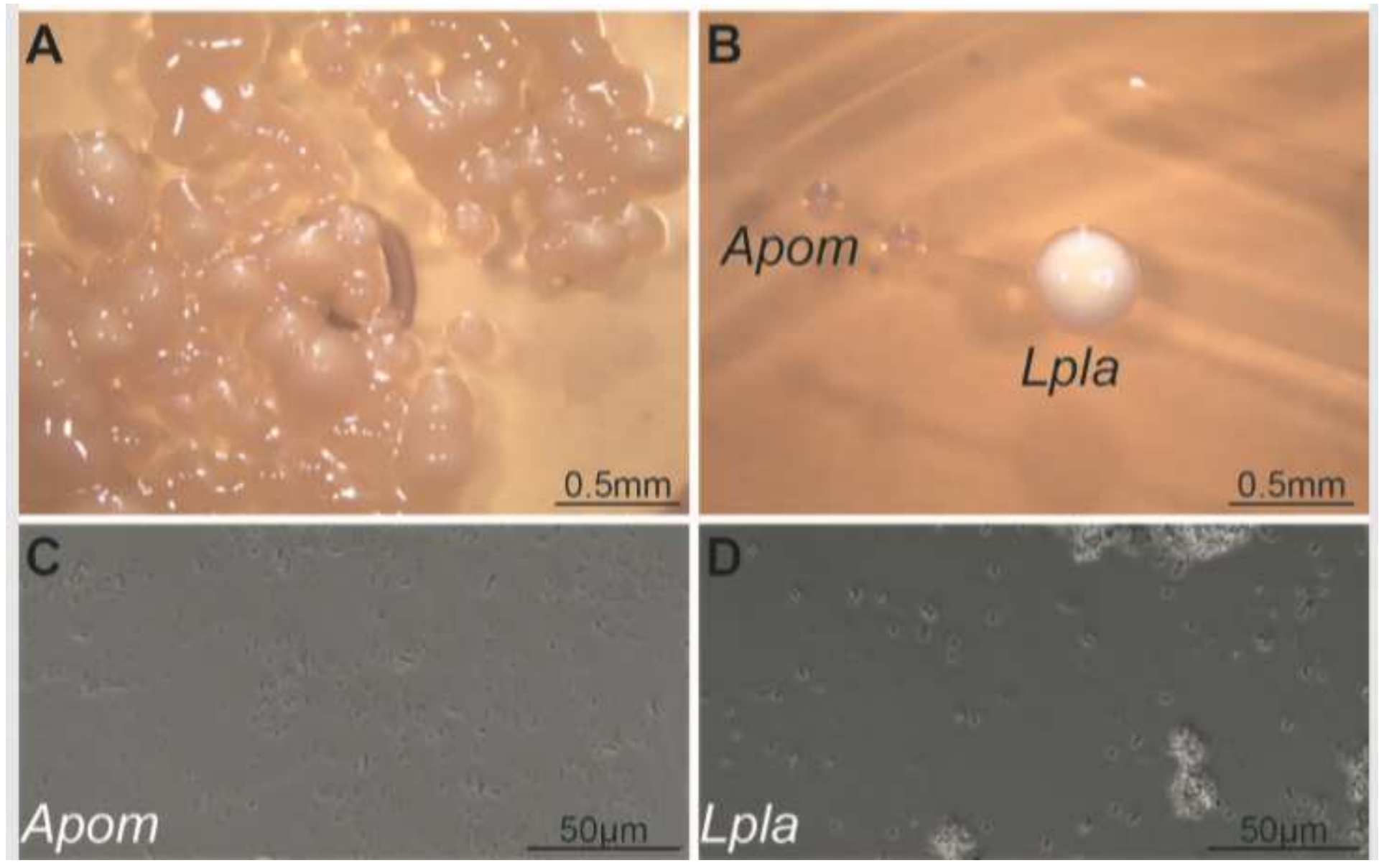

\section{Figure 1}

L. plantarum and A. pomorum are present on the surface of D. melanogaster. Upon streaking D. melanogaster on a plate, two types of heaps of bacteria were observed (A). We isolated single colonies that were white or beige (B). After sequencing the 16S rDNA, the white colonies were identified as $L$. plantarum (Lpla) and the beige colonies as A. pomorum Apom). Under the microscope, both bacteria are a rode shaped (L. plantarum, C, A. pomorum, D). 

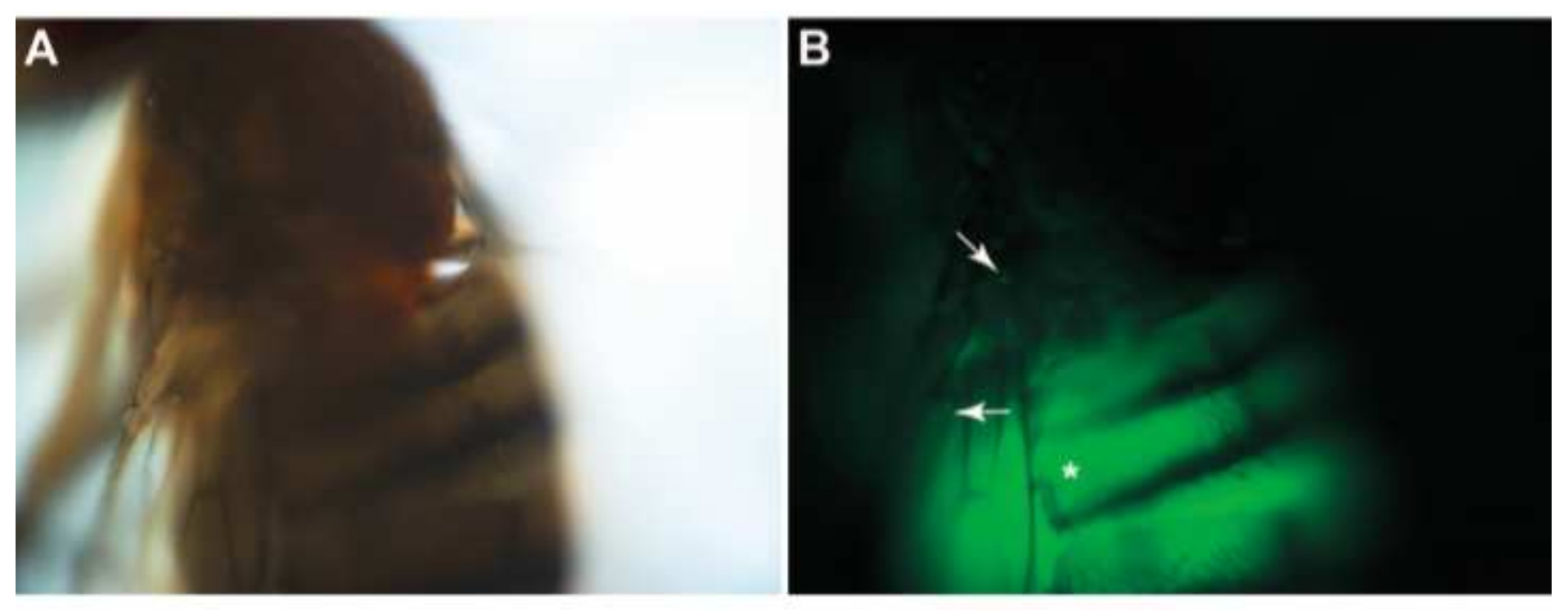

Figure 2

The ratio between $L$. plantarum and A. pomorum depends on the fly genotype. Applying qPCR, we detected L. plantarum and A. pomorum in the wash solution of fly surfaces in three independent experiments (1-3). In ebony mutant flies (ebonycc1 \& 3) that derive from Tübingen 2018 flies (Tü 2018) by gene editing (Crispr/Cas9), the L. plantarum to A. pomorum ratio is higher than in the original flies. The expression levels on Tübingen 2018 flies are set to one. Statistical analyses are not useful as the expression changes in the different experiments are enormous, although a clear trend is obvious.

\section{Supplementary Files}

This is a list of supplementary files associated with this preprint. Click to download.

- Figures1.jpg 